\title{
Persistent Increase in the Motivation to Take Heroin in Rats with a History of Drug Escalation
}

\author{
Serge H. Ahmed, Ph.D., John R. Walker, Ph.D., and George F. Koob, Ph.D.
}

The transition from stable to escalated levels of cocaine selfadministration has been shown to depend upon drug availability. The generality of this phenomenon is assessed here by studying the effects of availability on heroin selfadministration. Two groups of rats were trained on a 1-h continuous schedule of self-administration, after which, access to heroin (40 $\mu \mathrm{g} /$ injection) was increased to $11 \mathrm{~h}$ in one group (long access or $\operatorname{LgA}$ rats) or kept to $1 \mathrm{~h}$ in the other group (short access or ShA rats). After 18 sessions on this regimen, both ShA and $\operatorname{Lg} A$ rats were tested for extinction and stress-induced reinstatement of heroinseeking behavior. In $\mathrm{Lg} A$ rats, both total and first hour intake gradually escalated over time. After escalation, $\operatorname{Lg} A$ rats were slower to extinguish heroin-seeking behavior and responded more to the reinstating effect of stress after extinction. These findings show that: (1) the escalation process in drug consumption is common to both opiate and stimulant self-administration; (2) escalation in heroin consumption is associated with a persistent increase in the motivation for taking heroin.

[Neuropsychopharmacology 22:413-421, 2000] (C) 2000 American College of Neuropsychopharmacology. Published by Elsevier Science Inc.
KEY WORDS: Heroin; Cocaine; Self-administration; Homeostasis; Escalation; Set-point; Addiction; Relapse; Stress

A critical issue in the neurobiology of addiction is to explain the basis for the differences between controlled/ moderate and uncontrolled/excessive drug use, the latter being an essential feature of the addictive state (Koob and Le Moal 1997). The transition from drug use to addiction often involves a gradual process of escalation, whereby with chronic exposure to a drug, an indi-

From the Division of Psychopharmacology, Department of Neuropharmacology, The Scripps Research Institute, La Jolla, California.

Address correspondence to: S.H. Ahmed, Division of Psychopharmacology, Department of Neuropharmacology, CVN-7, The Scripps Research Institute, 10550 North Torrey Pines Road, La Jolla, CA 92037.

SHA and JRW contributed equally to the present work.

Received June 24,1999; revised September 23, 1999; accepted September 30, 1999. vidual's consumption becomes excessive and difficult to control (e.g., Gawin and Ellinwood 1989; Jaffe 1990; Lindesmith 1968; Marlatt et al. 1988; O'Brien et al. 1986). Because escalated use is common to many forms of drug addiction, the study of the factors that influence the initiation and maintenance of drug escalation may help in the understanding of the transition from controlled drug use to drug addiction.

Based on previous work using unlimited access to drug self-administration (Deneau et al. 1969; Johanson et al. 1976; Bozarth and Wise 1985), an animal model of the transition to increased drug intake was recently developed in the rat (Ahmed and Koob 1998). In this model, differential access to intravenous self-administration of cocaine produced two patterns of drug intake. With 1 hour of access per session (Short Access or ShA rats), drug intake remained low and stable over time. In contrast, with 6 hours of access per session (Long Access or LgA rats), drug intake gradually escalated to become significantly increased from baseline. Similarly, in 
humans, a sudden increase in drug availability can precipitate the transition to increased drug use (e.g., Gawin and Ellinwood 1989; Kramer et al. 1967; Wikler 1952). Although escalated cocaine use returned to pre-escalation levels after a forced abstinence of 1 month, it was reinstated to a level that was greater than that seen before abstinence. This increased vulnerability to relapse after a history of escalation may represent a factor contributing to the spiraling cycle of addiction (Ahmed and Koob 1998).

The present study was intended to assess the generality of these phenomena by studying the effects of increased availability on intravenous intake of another major drug of abuse, heroin. Both commonalities and differences already have been reported between the behavioral and biological effects of opiate and stimulant drugs (e.g., Ettenberg et al. 1982; Pettit et al. 1984). Prolonged access consisted of 11 hours access to heroin per session (LgA rats) and control access conditions consisted of 1 hour per session (ShA rats). Eleven hours were chosen, because it corresponds to a large portion of the night cycle and allows the experimenter to leave the animals in the operant boxes overnight. In addition, to determine the existence of eventual long-lasting changes in heroin-seeking behavior after escalation, both ShA and LgA groups were tested for extinction (25 sessions) and then for stress-induced reinstatement of heroin-seeking behavior (e.g., Ahmed and Koob 1997; Erb et al. 1996; Shaham and Stewart 1995).

\section{MATERIALS AND METHODS}

\section{Subjects}

Sixteen naive male Wistar rats (Charles River, Hollister, CA), weighing 330-450 $\mathrm{g}$ at the start of the behavioral experiment, were used. Upon their arrival in the laboratory, rats were housed in groups of two or three and maintained in a light- (12-h light-dark cycle; lights on at $6 \mathrm{AM})$ and temperature-controlled vivarium. All behavioral testing occurred during the dark phase of the light-dark cycle. Food and water were freely available, except when specified. All procedures were conducted in accordance with the National Institutes of Health Guide for the Care and Use of Laboratory Animals.

\section{Catheter Surgery and Patency}

Anesthetized rats (halothane-oxygen mixture, 1.0-1.5\% halothane) were prepared with catheters in the right jugular vein as described previously (Ahmed and Koob 1997). After surgery, catheters were flushed daily with $0.15 \mathrm{ml}$ of a sterile antibiotic solution containing heparinized saline (30 USP units $/ \mathrm{ml}$ ) and Timentin ${ }^{\circledR}(100$ $\mathrm{mg} / \mathrm{ml}$; SmithKline Beecham Pharmaceuticals, Philadelphia, PA). The patency of the catheter was checked by administering $0.15 \mathrm{ml}$ of the ultrashort-acting barbiturate anesthetic Brevital $\AA$ Sodium through the catheter ( $1 \%$ methohexital sodium, Eli Lilly, Indianapolis, IN) as described previously (Caine et al. 1993). Damaged catheters were repaired by inserting a short piece $(4-6 \mathrm{~mm})$ of a 26-gauge needle at the break point under halothane anesthesia. No rat had to be recatheterized in this study.

\section{Heroin Self-Administration}

Behavioral training was conducted in standard operant chambers (described in Ahmed and Koob 1997). Initially, rats were food restricted to maintain them at $85 \%$ of the body weight obtained with free-feeding conditions and trained to press a lever for $45-\mathrm{mg}$ food pellets on a continuous reinforcement schedule during 5-7 daily 30-min sessions. Two days after having been returned to an ad libitum food regimen, rats were catheterized and allowed to recover a minimum of 1 week before being given access to heroin. All rats were tested for heroin self-administration on a continuous reinforcement schedule for four successive phases (preescalation, escalation, extinction, and stress-induced reinstatement). Responding on the lever resulted in the delivery of one heroin injection over $4 \mathrm{sec}$ (3,6-diacetylmorphine $\mathrm{HCl}$, NIDA, dissolved in $0.1 \mathrm{ml}$ of sterile $\mathrm{NaCl}, 0.9 \%$ ) and initiated a 20 -sec time-out (TO) period signaled by a light cue located above the lever. No noncontingent injections of heroin were given. For the extinction and reinstatement phases, a second, but inactive, lever was added to control for possible nonspecific effects.

Pre-Escalation Phase. During this phase, rats were allowed to acquire IV self-administration of heroin. All animals were allowed access to a FR1: TO 20-sec schedule of heroin self-administration with 1 -h access to heroin for nine consecutive sessions ( $20 \mu \mathrm{g}$ per infusion).

Escalation Phase. This phase started the day after the end of the pre-escalation phase. The dose of heroin was increased to $40 \mu \mathrm{g}$ per infusion, so that overnight intake in LgA rats did not exceed the volume of the syringe (i.e., $10 \mathrm{ml}$ ). Two balanced groups of rats with the same mean body weight and the same rate of heroin selfinfusions, as measured during the last three sessions of the pre-escalation phase, were formed. During the escalation phase, groups differed only with respect to heroin availability. In one group, drug access was increased to 11 hours per session (Long Access or LgA group). This duration was chosen, because it corresponds to a large portion of the night cycle and allows the experimenter to leave the animals in the operant boxes overnight. In the other group (Short Access or ShA group), drug access time was kept to 1 hour as during the pre-escalation phase. Food and water were 
freely available in the operant boxes throughout the escalation phase.

Extinction Phase. Two weeks after the last session of escalation, rats were subjected to extinction. During each daily 1-h extinction session, the levers were extended, but pumps were turned off so that presses on the active lever resulted only in the presentation of the light TO cue previously associated with heroin infusions. Presses on the inactive lever had no scheduled consequence. Moreover, throughout extinction, rats were not connected to the infusion system. This procedure was intended to equalize the conditions of extinction with those to be used during testing sessions.

Reinstatement Phase. Following 25 sessions of extinction, rats were given five control testing sessions and one footshock testing session. During the control testing sessions, the typical 1-h extinction session was preceded by a 15 -min period in the operant box with no footshock delivered while during the footshock testing session, the session was preceded by 15-min of intermittent footshock. During both testing sessions, the levers were extended, heroin was not available, and rats were not connected to the infusion system to avoid potential damaging effects resulting from the jumping behaviors induced by footshock stress.

\section{Electric Footshock}

The parameters of the footshock were selected based on what was used in a previous study (Shaham and Stewart 1995). Footshocks (alternating current; $1 \mathrm{~mA} ; 0.5 \mathrm{~s}$ trains) were delivered through a scrambler (Coulbourn Instruments, Allentown, PA) to the grid floor of the operant chamber. They were administered during a period of 15-min in an intermittent manner according to a variable interval schedule (mean interval: $40 \mathrm{~s}$; range: 10-70 s).

\section{Data Analysis}

Data obtained during the escalation and extinction phases were subjected to two-way analyses of variance (ANOVA) with one between-factor, experimental groups (ShA versus LgA group), and one within-factor, sessions. The data obtained during drug-loading (that is, the first hour of access to heroin) were subjected to a two-way ANOVA with two within-factors, sessions of self-administration (before vs. after escalation) and time course of drug self-infusions within the first hour $(3 \times$ 20 -min blocks). Changes in the hourly pattern of heroin intake after escalation were assessed using a two-way ANOVA with two within-factors, sessions of selfadministration (first vs. last session of the escalation phase) and time course of self-infusions during 11 hours $(11 \times 1$-h blocks). Post hoc comparisons for inter- actions were carried out by tests for simple main effects. For both the extinction and reinstatement phases, the total number of responses (TO responses included) served as the dependent variable. The inclusion of TO responses in the performance is justified, because during both extinction and testing sessions, all of the responses emitted were nonreinforced. In addition, separate analyses performed on either the first responses that initiated the TO period or those produced during it yielded a similar statistical outcome during testing sessions. In the course of the self-administration study, which lasted almost 4 months, three rats died at different times during extinction (1 ShA and 2 LgA rats). In addition, 1 ShA rat was excluded from the study because: (1) it excessively responded during time-out periods; (2) it chewed the metal spring, on some occasions, damaging the tubing for heroin delivery; and (3) it was a statistical outlier for heroin intake (more than 2 SD above the mean). Six ShA and 6 LgA rats remained for analysis. Because of the relatively small samples $(n=$ 6 per group) and the high interindividual variability observed in response to footshock stress, data obtained during the reinstatement phase were analyzed with the nonparametric Mann-Whitney U test (Siegel 1956).

\section{RESULTS}

\section{Escalation in Heroin Self-Administration}

Figure 1 depicts the course of escalation in total heroin intake following increased heroin availability. In contrast to ShA rats, for which heroin intake remained low and stable $\left(\mathrm{F}_{(17,170)}=0.02\right)$, LgA rats progressively increased their consumption over time from 35 to an average of 64 infusions during the last session $\left(\mathrm{F}_{(17,170)}=\right.$ $17.26, p<.01)$. Post hoc comparisons revealed that heroin intake in LgA rats significantly increased above the level of the 1st session from the seventh session of escalation onward (simple main effect, $p$ s $<.05$ ). As shown in Figure 2 the hourly pattern of heroin intake followed a characteristic temporal course: high in the first hour, heroin intake stabilized later at a lower level, ruling out any within-session tolerance. In LgA rats after escalation, heroin intake increased at all hours (first versus last session, $\left.\mathrm{F}_{(1,5)}=19.49, p<.01\right)$.

To further analyze the nature of escalation, intake during the first hour was compared between groups (Figure 3). Heroin intake was virtually identical between groups at the beginning of the escalation phase. However, heroin intake in LgA rats progressively increased above the level of intake observed in ShA rats (group $\times$ session interaction: $\mathrm{F}_{(17,170)}=5.01, p<.01$ ). Significant differences between groups were detected from the seventh session of escalation onward (simple main effect, Fs $>5.10, p s<.05$ ), except during session 14. It is important to note that the increased heroin con- 


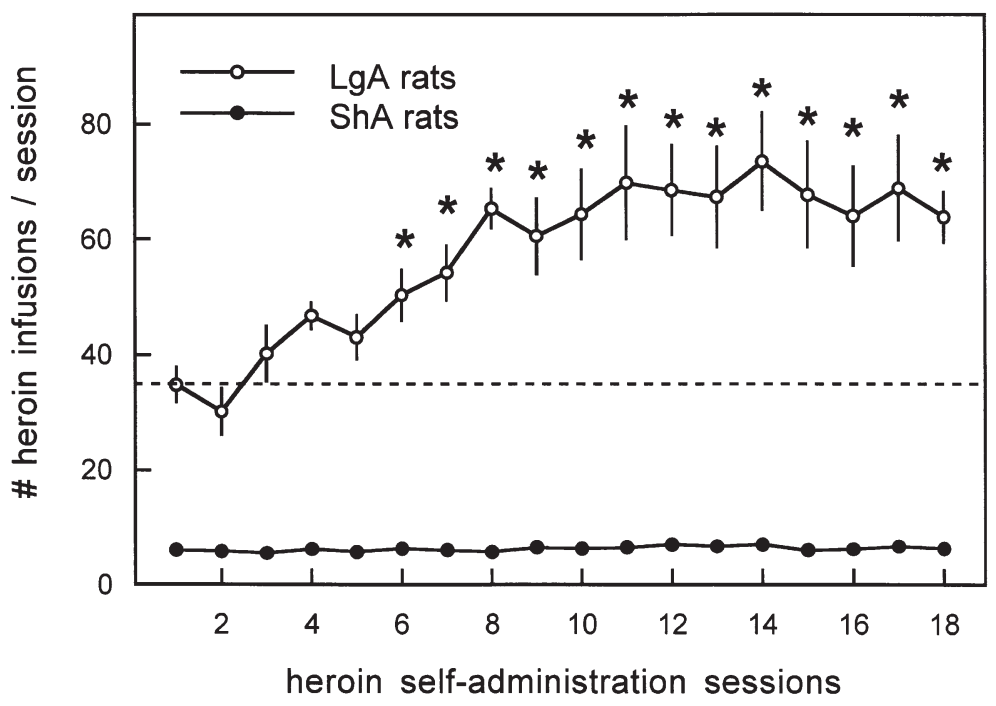

Figure 1. Effect of drug availability on total intravenous heroin self-infusions (mean \pm SEM). During the escalation phase, rats had access to heroin (40 $\mu$ g per infusion) for 1 hour (ShA rats, $n=$ 6) or 11 hours per session (LgA rats, $n=6$ ). Regular 1-hour (ShA rats) or 11-hour (LgA rats) sessions of heroin self-administration were performed 6 days a week. The dotted line indicates the mean ( \pm SEM) number of heroin selfinfusions of LgA rats during the first 11-hour session. ${ }^{*} p<.05$, different from the first session (paired $t$-test). sumption was observed for the entire 11-hour session after escalation and was not confined to the first hour (see Figure 2). Escalated heroin intake is not attributable to nonspecific behavioral effects related to heroin intoxication, because no group differences were observed in TO responses during the last six sessions of the escalation phase, where the difference between groups in heroin intake was well established (group factor: $\mathrm{F}_{(1,10)}=0.08$; group $\times$ session interaction: $\mathrm{F}_{(5,50)}=$ 1.78; data not shown).

Typically, rats take more infusions with short interinfusion intervals early in the self-administration session, a phenomenon true for both stimulants and opiates (Pickens et al. 1978). This drug-loading behavior

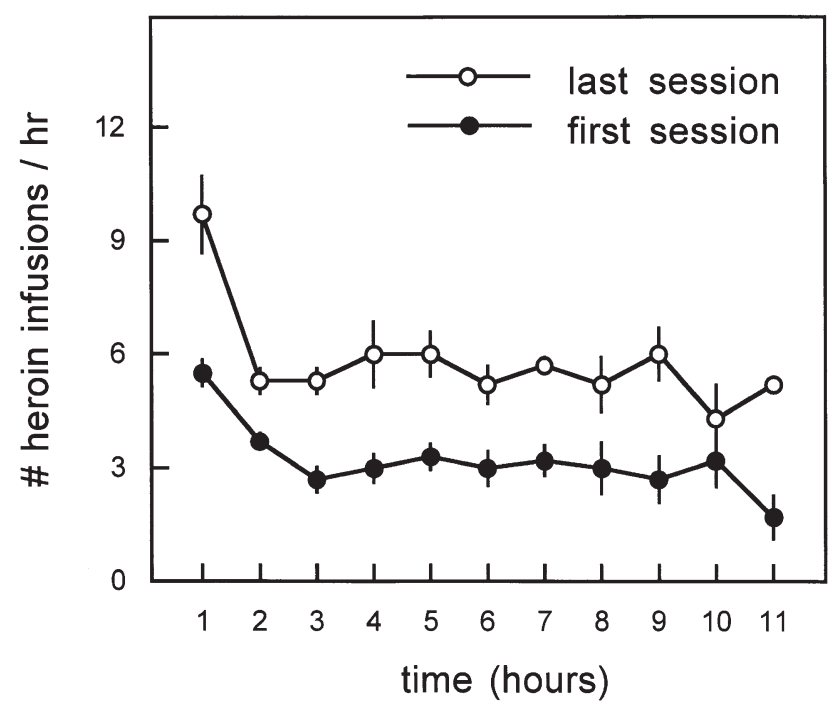

Figure 2. Change in the pattern of intravenous heroin selfadministration of $\operatorname{LgA}$ rats after escalation $(n=6)$. Points represent the mean ( \pm SEM) number of heroin self-infusions per hour during the first and last 11-hour sessions of the escalation phase. marks the transition from the nondrugged to the intoxicated state. To assess possible changes in drug-loading, the temporal distribution of heroin self-infusions within the first hour was compared before and after escalation (Figure 4). Drug-loading was manifested by a higher rate of self-infusions during the first $20 \mathrm{~min}$, as compared to the rest of the session (ShA rats: $\mathrm{F}_{(2,10)}=$ 18.91, $p<.01 ; \mathrm{LgA}$ rats: $\left.\mathrm{F}_{(2,10)}=33.94, p<.01\right)$. After escalation, drug-loading increased in $\operatorname{LgA}$ rats $\left(\mathrm{F}_{(1,5)}=\right.$ 22.81, $p<.01)$; no change was observed in ShA rats $\left(\mathrm{F}_{(1,5)}=0.62\right)$.

Extinction and Reinstatement of Heroin-Seeking Behavior. Figure 5 depicts the total number of responses (including TO responses) on the active and inactive levers during extinction sessions. As expected, presses on the active lever decreased progressively during extinction $\left(\mathrm{F}_{(24,240)}=12.34, p<.01\right)$. Extinction of heroin-seeking behavior was, however, slower in LgA rats compared to ShA rats (group $\mathrm{x}$ session interaction: $\left.\mathrm{F}_{(24,240)}=2.48, p<.01\right)$. Post hoc comparisons revealed that $\operatorname{LgA}$ rats responded on the active lever significantly more than ShA rats during sessions 2, 3, 4, 5, 9, and 10 (Fs $>5.38, p<.05)$; the difference between groups was close to significance during session one $\left(\mathrm{F}_{(1,24)}=4.47, p=.060\right)$. Presses on the inactive lever, which were very low, decreased also during extinction $\left(\mathrm{F}_{(24,240)}=9.40, p<.01\right)$. There was, however, no difference between groups in inactive performance (group factor: $\mathrm{F}_{(1,10)}=0.34$; group $\times$ session interaction: $\mathrm{F}_{(24,240)}=$ $0.81)$.

Figure 6 depicts the total number of presses on the active lever during both the control and footshock testing sessions. Control performance was expressed as the mean of the last three control testing sessions during which responding was very stable and not different between groups (active responding: $U=13, p=0.24$; inactive responding: $U=10, p=.12$ ). Footshock stress in- 


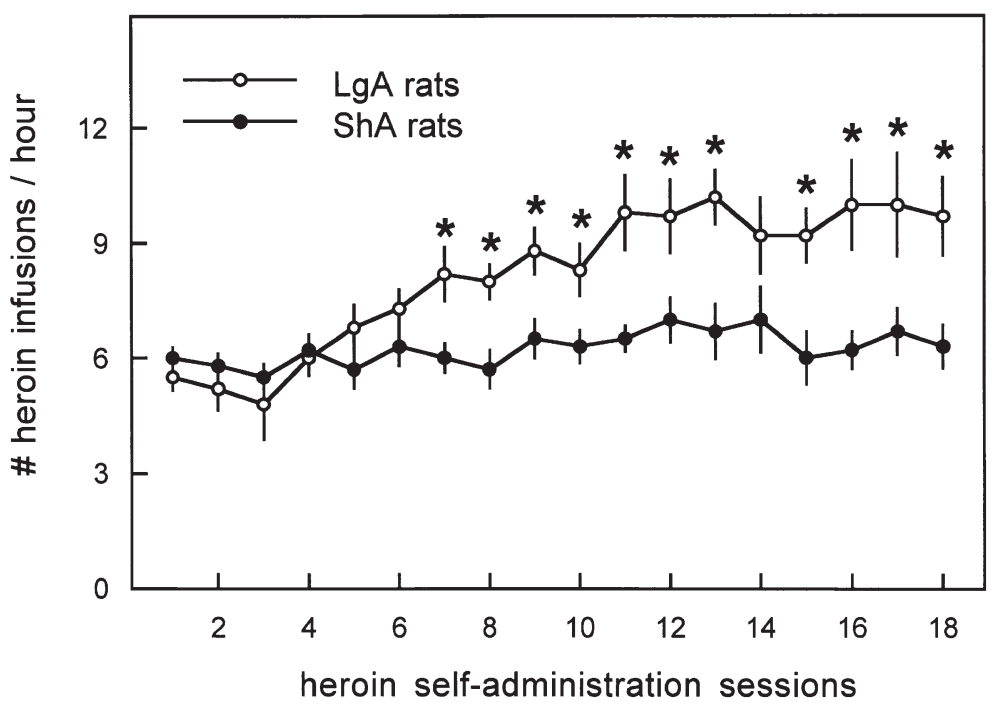

Figure 3. Effect of drug availability on first-hour heroin self-infusions (mean \pm SEM). During the escalation phase, rats had access to heroin (40 $\mu \mathrm{g}$ per infusion) for 1 hour (ShA rats, $n=6$ ) or 11 hours per session (LgA rats, $n=6$ ). Regular 1-hour (ShA rats) or 11-hour (LgA rats) sessions of heroin self-administration were performed 6 days a week. ${ }^{*} p<.05$, different from ShA rats (simple main effect). creased only active responding, and this effect was more pronounced in LgA rats than in ShA rats $(\mathrm{U}=8$, $p=.06)$. There was no difference between groups in responding on the inactive lever after footshock $(U=12$, $p=.19)$. The relatively high variability observed in inactive responding in both groups was attributable to two rats (one ShA and one LgA rat) that also seemed to reinstate responding on the inactive lever.

\section{DISCUSSION}

The present study demonstrates the existence of two distinct patterns of heroin-taking behavior, one characterized by a stable and low intake, the other by an escalating intake. The transition from stable/low to escalat- ing heroin intake depended upon the amount of drug exposure. These observations extend previous observations by the same laboratory on cocaine self-administration and indicate that the escalation process is common to both opiate and stimulant drugs. After escalation, rats with a history of heroin escalation were slower to extinguish heroin-seeking behavior and responded more to the reinstating effect of stress after a prolonged period of extinction.

\section{Transition from Moderate to Increased Heroin Use: Change in Heroin Set Point?}

The transition to increased levels of drug intake has been hypothesized to result from the gradual fixation of a new set point for the regulation of drug self-adminis-
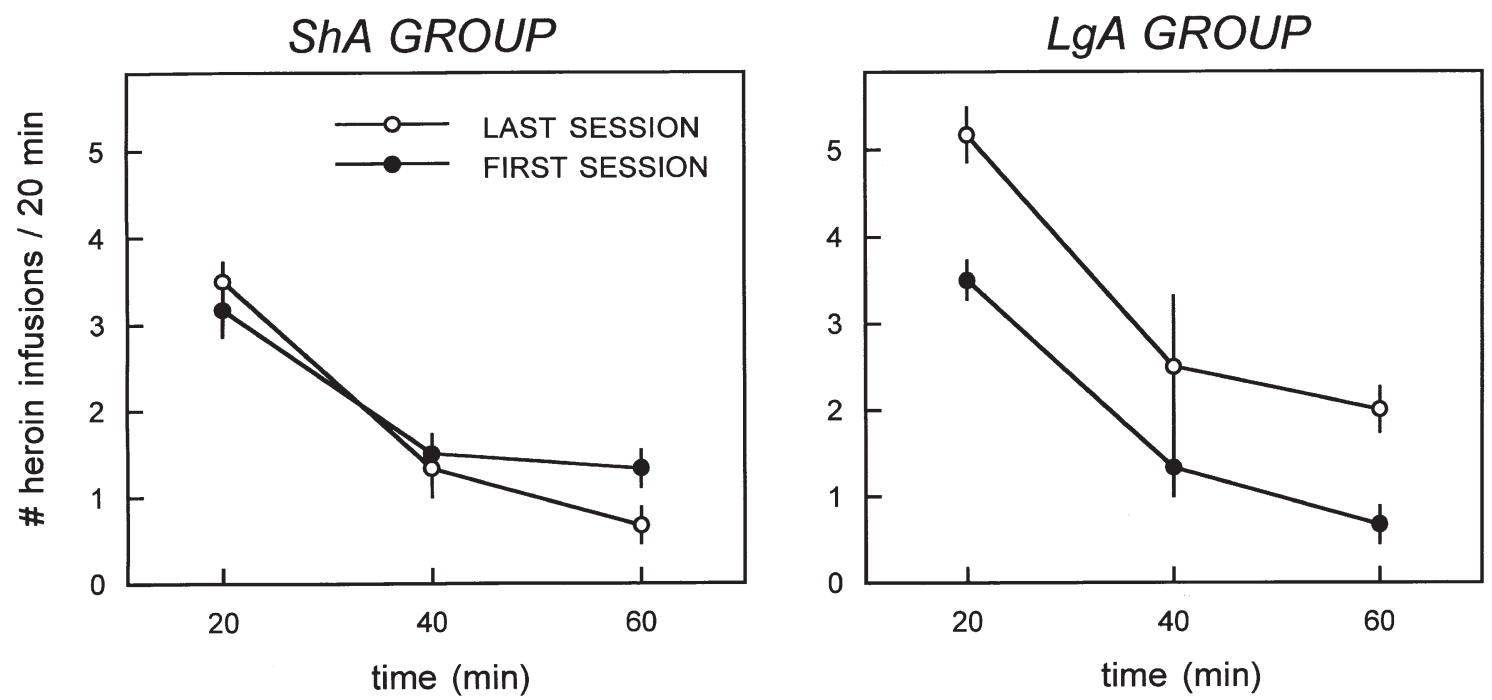

Figure 4. Change in heroin-loading behavior after escalation. Points represent mean ( \pm SEM) number of intravenous heroin self-infusions ( $40 \mu \mathrm{g}$ per infusion) during the first hour of the first and last sessions of the escalation phase (left panel: ShA rats, $n=6$; right panel: LgA rats, $n=6$ ). 

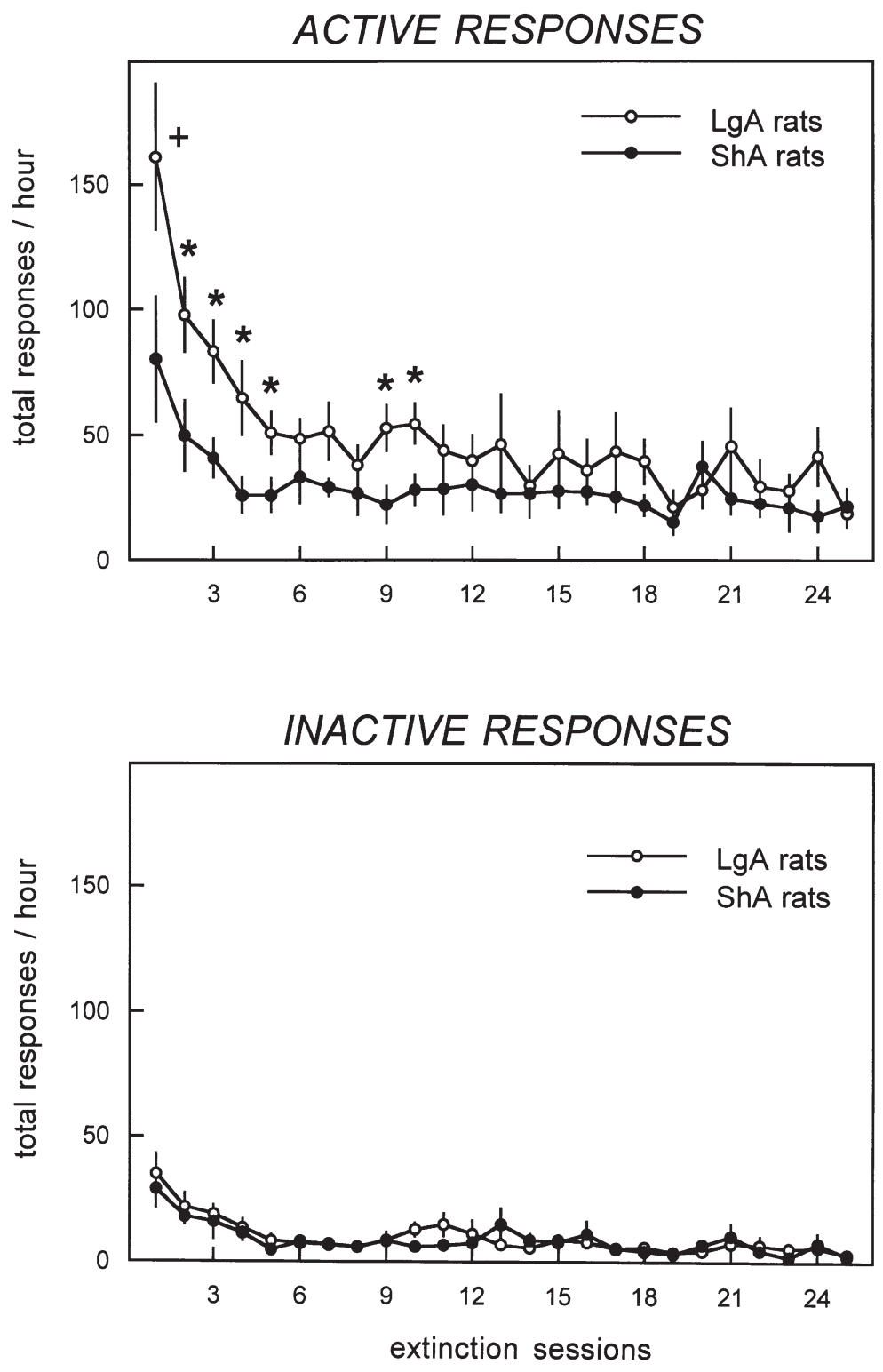

Figure 5. Extinction of heroin-seeking behavior in both ShA $(n=6)$ and LgA rats $(n=6)$ after escalation. Each point represents the mean $( \pm$ SEM) total responses on the active (upper panel) and inactive lever (lower panel) in a 1-hour session of extinction (25 sessions in total). During extinction, lever presses were without consequences. $+p=.06,{ }^{*} p<$ .05 , different from ShA rats (simple main effect). tration. A major function of a drug set point would be to limit drug consumption at a certain level (Ahmed and Koob 1998; 1999). That is, as with known physiological set points (e.g., for body weight or temperature regulation), the set point for drug self-administration would serve as an internal template against which deviations in drug effects are detected and are eventually corrected by an appropriate response in order to maintain these effects at a preferred level. The existence of a set point for the regulation of drug self-administration is supported by two lines of evidence. First, with limited access time to a continuous schedule of self-administration, subjects tend to limit their consumption to a certain level that is very stable between self-administration sessions spontaneously. This phenomenon is true for both stimulants and opiates (for reviews, Pickens et al. 1978; Yokel 1987). Second, rate of self-injections and dose per injection are inversely related: as the dose per injection decreases, the rate of self-injections increases (for reviews, Pickens et al. 1978; Yokel 1987). It has been recently demonstrated that rats adjust their self-injection rate to virtually maintain the same level of cocaine effects across a wide range of doses. Thus, twofold, fourfold, and eightfold changes in cocaine dose were compensated by equivalent changes in self-infusion rate (Ahmed and Koob 1999).

The hypothesis that the transition to increased levels of intake is mediated by a change in drug set point is supported by recent data obtained in rats self-administering cocaine (Ahmed and Koob 1998; 1999). Thus, after an initial period of escalation, cocaine intake by LgA rats stabilized for several weeks at a level two times higher than before escalation. Furthermore, the doseeffect function for cocaine self-administration was 

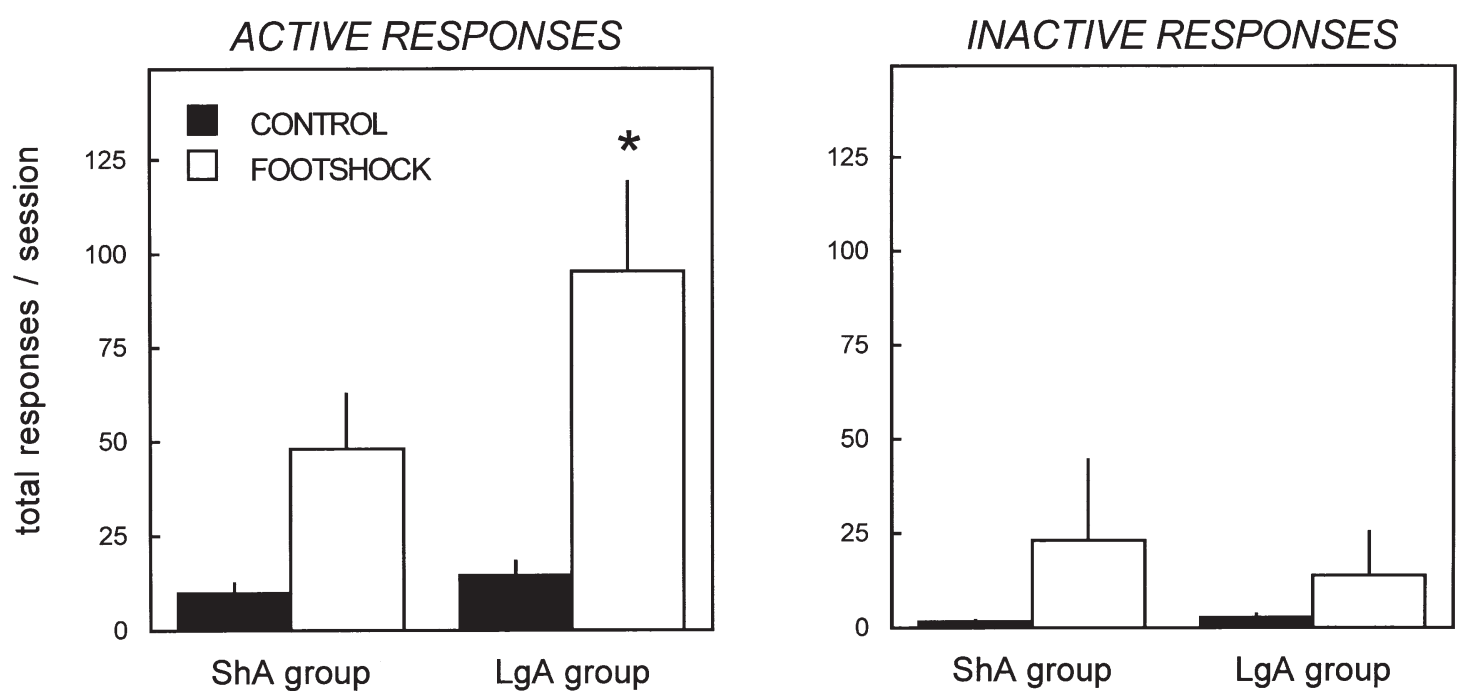

Figure 6. Effect of intermittent footshock on heroin-seeking behavior after extinction. Left Panel. Each bar represents the mean $( \pm$ SEM) total presses on the active lever in a 1-hour extinction session following a 15-min shock-free period (control testing session) or 15-min of intermittent footshock ( $1 \mathrm{~mA} ; 0.5 \mathrm{sec}$ on, with a mean off period of $40 \mathrm{sec})$ in both ShA and $\mathrm{LgA}$ rats. During these testing sessions, presses were without consequences. Right Panel. Same, but for presses on the inactive lever. ${ }^{*} \mathrm{p}=.06$, different from ShA rats (Mann-Whitney $U$ test).

shifted upward in LgA rats, with no change in the minimum effective dose suggesting that escalated levels of intake are probably not the result of a simple change in sensitivity to the drug effects (i.e., tolerance/sensitization; for a full discussion of this important issue, see Ahmed and Koob 1999). In the present study, after a period of gradual escalation, escalated heroin intake also stabilized between sessions at a level nearly two times greater than before escalation. Furthermore, after escalation, heroin consumption stabilized within a session at a level higher than before escalation (see Figure 2). These phenomena suggest that, as with cocaine, a change in the set point for heroin self-administration could be responsible for the transition to increased levels of heroin intake. To confirm this hypothesis fully, however, it will be necessary to verify whether the dose-response curve for heroin self-administration also is shifted upward after escalation. Nonetheless, the remarkable similarities between the dynamic of the escalation process for cocaine and for heroin self-administration suggest that the factors responsible for the transition to increased levels of drug intake are common to both stimulant and opiate drugs.

In the present study, escalation in heroin intake was produced by increasing availability from 1 to 11 hours. This duration was essentially based on convenience. It will be important for future studies to determine whether similar results will be obtained with shorter periods of availability, as with escalation in cocaine intake that was observed after increasing availability to 6 hours. In addition to establishing full comparability between escalation in opiate and stimulant intake, these studies will determine whether the rate of escalation is a function of the duration of access to drug self-administration.

\section{Long-Lasting Effects of a History of Drug Escalation: Implications for Relapse}

A high rate of relapse is commonly observed following successful short-term or even long-term abstinence (Jaffe 1990; McLellan et al. 1992; Vaillant 1992). In this context, understanding the factors contributing to relapse constitutes an important step for the development of treatment strategies. To date, several events have been shown to trigger reinstatement of drug-seeking behavior after extinction. Generally, these events are analogous to those supposed to trigger relapse in drug addicts (i.e., exposure to a stressor, a drug, or stimuli previously associated with drug use, and spontaneous withdrawal in opiate-dependent individuals; see for example, Carroll 1985; Davis and Smith 1976; de Wit and Stewart 1981; Gerber and Stretch 1975; Shaham and Stewart 1995; Shaham et al. 1996). Unknown, however, are the factors that could influence the response of the individual to a given relapsing event. The present study suggests that a history of drug escalation may increase the vulnerability to stress-induced reinstatement of drug-seeking behavior.

First, LgA rats showed an increase in drug-seeking behavior during extinction compared to ShA rats. This increase in extinction performance persisted for several days and was not attributable to nonspecific effects, because both groups responded similarly on the inactive lever. Only a few studies have attempted to investigate the factors influencing extinction performance. Recent 
studies of nondrug reinforcement indicate that, at an early stage of operant training, extinction performance is determined mostly by the incentive value of the outcome for which the animals respond (e.g., food pellets), but is relatively insensitive to their current motivational state (e.g., hungry versus sated). However, over repeated training sessions, extinction performance becomes mainly automatic and relatively insensitive to the incentive value of the reinforcer (for reviews, Dickinson 1994; Dickinson and Balleine 1994). At this stage, extinction performance would become more sensitive to the current motivational state of the individual. Indeed, food-deprivation recently has been shown to increase extinction responding in rats overtrained in a continuous schedule of food reinforcement (i.e., 16 daily 40-min sessions of operant training, Ahmed and Koob 1997). In this context, the increased extinction performance observed in $\mathrm{LgA}$ rats could reflect an increase in the motivation to obtain heroin.

Second, after a prolonged period of extinction and abstinence, $\operatorname{LgA}$ rats seemed to be more susceptible to the reinstating effect of an aversive stressor. Recently, it has been speculated that a stressor may trigger reinstatement of drug-seeking behavior by providing some internal cues that predict drug availabilty (Ahmed and Koob 1997; for a similar idea, see Carroll 1985). According to this account, the motivation for drug-taking does not dissipate during extinction, but its behavioral expression progressively becomes inhibited by information of nonreinforcement. The reappearance after extinction of cues predictive of drug availability would have the effect of removing this inhibition by setting the occasion for responding, revealing in this way the "latent" motivation for taking the drug (see also, Bouton and Swartzentruber 1991; Bouton 1993; Swartzentruber 1995). This analysis is consistent with accounts of extinction phenomena in terms of conditioned inhibition (e.g., Pavlov 1927; Konorski 1967; Rescorla 1969) and with a recent finding showing that a reversible inactivation of the septum, a brain area involved in behavioral inhibition, also triggers reinstatement of heroin-seeking behavior after extinction (Shaham et al. 1998). In this regard, the increased response to stress observed in LgA rats would reflect their increased motivation to obtain heroin, confirming the above analysis of the extinction data.

In summary, the present study shows that the transition to increased levels of heroin intake is produced by an increase in drug availabilty. This observation confirms and extends previous findings obtained in cocaine self-administration. Some features of the escalation process in heroin intake (stabilization between- and withinsession) suggests that a change in the set point for the regulation of heroin self-administration would be responsible for the transition to increased levels of intake. Furthermore, rats that have previously developed an increased intake of heroin were subsequently slower to extinguish heroin-seeking behavior during the first week of extinction and seemed to be more prone to relapse after stress. Altogether, these data suggest that the change in heroin set point produced by increasing drug availability also is associated with a persistent increase in the motivation for taking heroin.

\section{ACKNOWLEDGMENTS}

This is publication number 12556-NP from The Scripps Research Institute. This work was supported by Grant DA04043 from the National Institute on Drug Abuse (GFK). The authors thank Robert Lintz for his invaluable technical assistance, Tanya Joswig for her help in conducting part of the experiment reported, and Mike Arends for his correction of the remaining errors.

\section{REFERENCES}

Ahmed SH, Koob GF (1997): Cocaine- but not food-seeking behavior is reinstated by stress after extinction. Psychopharmacology 132:289-295

Ahmed SH, Koob GF (1998): Transition from moderate to excessive drug intake: Change in hedonic set point. Science 282:298-300

Ahmed SH, Koob GF (1999): Long-lasting increase in the set point for cocaine self-administration after escalation in rats. Psychopharmacology 146:303-312

Bouton ME, Swartzentruber D (1991): Sources of relapse after extinction in Pavlovian and instrumental learning. Clin Psychol Rev 11:123-140

Bouton ME (1993): Context, time, and memory retrieval in the interference paradigms of Pavlovian learning. Psychol Rev 114:80-99

Bozarth MA, Wise RA (1985): Toxicity associated with longterm intravenous heroin and cocaine self-administration in the rat. J Am Med Assoc 254:81-83

Caine SB, Lintz R, Koob GF (1993): Intravenous drug-selfadministration techniques in animals. In Sahgal A (ed), Behavioural Neuroscience: A Practical Approach, vol. 2. New York, Oxford University Press, pp 117-143

Carroll ME (1985): The role of food deprivation in the maintenance and reinstatement of cocaine-seeking behavior in rats. Drug Alcohol Depend 16:95-109

Davis WM, Smith SG (1976): Role of conditioned reinforcers in the initiation, maintenance, and extinction of drugseeking behavior. Pavlov J Biol Sci 11:222-236

de Wit H, Stewart J (1981): Reinstatement of cocaine-reinforced responding in the rat. Psychopharmacology 75:134-143

Deneau G, Yanagita T, Seevers MH (1969): Self-administration of psychoactive substances by the monkey. Psychopharmacologia 16:30-48

Dickinson A (1994): Instrumental conditioning. In Mackintosh NJ (ed), Animal Learning and Cognition (Series 
title: Handbook of Perception and Cognition, vol. 3). San Diego, Academic Press, pp 45-79

Dickinson A, Balleine B (1994): Motivational control of goaldirected action. Anim Learn Behav 22:1-18

Erb S, Shaham Y, Stewart J (1996): Stress reinstates cocaineseeking behavior after prolonged extinction and a drugfree period. Psychopharmacology 128:408-412

Ettenberg A, Pettit HO, Bloom FE, Koob GF (1982): Heroin and cocaine intravenous self-administration in rats: Mediation by separate neural systems. Psychopharmacology 78:204-209

Gawin FH, Ellinwood EH (1989): Cocaine dependence. Annu Rev Med 40:149-161

Gerber GJ, Stretch R (1975): Drug-induced reinstatement of extinguished self-administration behavior in monkeys. Pharmacol Biochem Behav 3:1055-1061

Jaffe JH (1990): Drug addiction and drug abuse. In Gilman AG, Rall TW, Nies AS, Taylor P (eds), Goodman \& Gilman's The Pharmacological Basis of Therapeuthics (8th ed.). New York, Pergamon Press, pp 522-573

Johanson CE, Balster RL, Bonese K (1976): Self-administration of psychomotor stimulant drugs: The effects of unlimited access. Pharmacol Biochem Behav 4:45-51

Konorski J (1967): Integrative Activity of the Brain: An Interdisciplinary Approach. Chicago, University of Chicago Press

Koob GF, Le Moal M (1997): Drug abuse: Hedonic homeostatic dysregulation. Science 278:52-58

Kramer JC, Fischman VS, Littlefield DC (1967): Amphetamine abuse: Pattern and effects of high doses taken intravenously. J Am Med Assoc 201:305-309

Lindesmith AR (1968): Addiction and opiates. Chicago, Aldine

Marlatt GA, Baer JS, Donovan DM, Kivlahan DR (1988): Addictive behaviors: Etiology and treatment. Ann Rev Psychol 39:223-252

McLellan AT, O’Brien CP, Metzger D, Alterman AI, Cornish J, Urschel $H$ (1992): How effective is substance abuse treatment-compared to what? In O'Brien CP, Jaffe JH (eds), Addictive States. New York, Raven Press, pp 231-252

O'Brien CP, Ehrman R, Ternes JW (1986): Classical conditioning in human opioid dependence. In Goldberg SR,
Stolerman IP (eds), Behavioral analysis of Drug Dependence. Orlando,FL: Academic Press, pp 329-356

Pavlov IP (1927): Conditioned Reflexes: An Investigation of the Physiological Activity of the Cerebral Cortex. London: Oxford University Press

Pettit HO, Ettenberg A, Bloom FE, Koob GF (1984): Destruction of dopamine in the nucleus accumbens selectively attenuates cocaine but not heroin self-administration in rats. Psychopharmacology 84:167-173

Pickens R, Meisch RA, Thompson T (1978): Drug self-administration: An analysis of the reinforcing effects of drugs. In Iversen LL, Iversen SD, Snyder SH (eds), Affective Disorders: Drug Actions in Animals and Man (Series title: Handbook of Psychopharmacology, vol. 14). New York, Plenum Press, pp 1-37

Rescorla RA (1969): Pavlovian conditioned inhibition. Psychol Bull 72:77-94

Shaham Y, Stewart J (1995): Stress reinstates heroin-seeking in drug-free animals: An effect mimicking heroin, not withdrawal. Psychopharmacology 119: 334-341

Shaham Y, Rajabi H, Stewart J (1996): Relapse to heroinseeking in rats under opioid maintenance: The effects of stress, heroin priming, and withdrawal. J Neurosci 16:1957-1963

Shaham Y, Leung S, McDonald RJ, Stewart J (1998): Disruption of inhibitory processes may be involved in stressinduced relapse to heroin. Soc Neurosc Abstr 24:498

Siegel S (1956): Nonparametric Statistics for the Behavioral Sciences. New York: McGraw-Hill

Swartzentruber D (1995): Modulatory mechanisms in Pavlovian conditioning. Anim Learn Behav 23:123-143

Vaillant GE (1992): Is there a natural history of addiction? In O'Brien CP, Jaffe JH (eds), Addictive States. New York, Raven Press, pp 41-57

Wikler A (1952): A psychodynamic study of a patient during self-regulated readdiction to morphine. Psychiat Quart 26:270-293

Yokel RA (1987): Intravenous self-administration: Response rate, the effects of pharmacological challenges, and drug preference. In Bozarth MA (ed), Methods of Assessing the Reinforcing Properties of Abused Drugs. New York, Springer-Verlag, pp 117-141 